\title{
Speckle pattern formation in spatially limited optical systems
}

\author{
M.M. Kotov, V.N. Kurashov, A.A. Goloborodko \\ Optical Processing Laboratory, Faculty of Radiophysics, Electronics and Computer Systems, \\ Taras Shevchenko National University of Kyiv, \\ 64, Volodymyrska str., 01033 Kyiv, Ukraine
}

\begin{abstract}
The dependences of statistical parameters inherent to speckle patterns on the object roughness and aperture size have been investigated. The experimental results that confirm theoretical dependence quality within the limits of errors were obtained. It has been shown that spatial finiteness of the optical system causes significant changes of transferred field.
\end{abstract}

Keywords: speckle, Fourier optics, aperture effects, heterogeneous objects.

Manuscript received 28.10.15; revised version received 27.01.16; accepted for publication 16.03.16; published online 08.04.16.

\section{Introduction}

Speckles play an important role in many fields where optical radiation is transmitted by or reflected from objects with a wavelength-scale roughness [1]. Important cases include determining the size of small particles by using the dynamic light scattering method [2], synthetic-aperture radar imagery in the microwave region [1], ultrasound medical imagery of organs of human body [3], optical coherence monitoring laserbased techniques are a promising tool for studying cell activity [4], etc. However, random wave phase distortions often occur when the tested object is illuminated or wave propagates through the measuring channel. This fact was noticed in [5], where results of diffuse surface testing were significantly regressed because of the speckle patterns in the registration area of the Shack-Hartmann sensor (Fig. 1). The main reason of the errors is both deformation of Fourier image of the forming lens aperture in the CCD matrix plane in the presence of phase heterogeneities and random centroid offset because of fluctuations of the speckle pattern field intensity. Obviously, the determinant factor that describes these effects is the average spot size of the speckle pattern. It is considered that the spot size in the Fourier plane depends on the aperture of the forming lens diaphragm only and does not depend on the statistical characteristics of the diffuse surface, in particular, on relief correlation properties [1]: $\varepsilon \sim \lambda z / a$, where $\lambda$ is the wavelength, $z$ - distance between the object and the observation plane, $a-$ size of the illuminated aperture. This approach is valid when the spatial spectrum of the optical field is wide enough, at least, it is much wider than the spectral function of the aperture. However, this claim is fundamentally wrong for the spatially limited optical system. Therefore, the statistical characteristics of speckle patterns must be defined by the correlation properties of phase distortion of the field. Thus, investigation of the influence of spatial finiteness of optical system on properties of the transferred speckle field is the purpose of this paper.

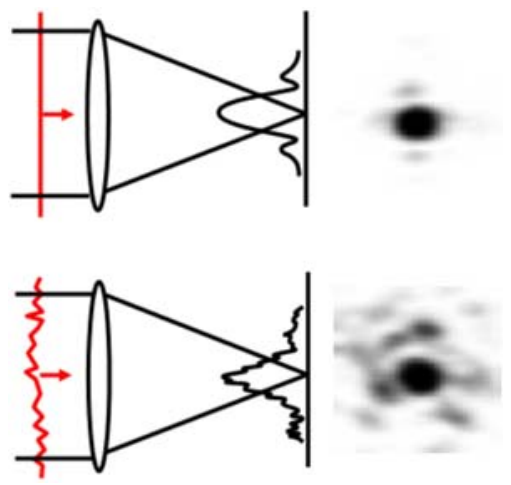

Fig. 1. Influence of small phase fluctuations on the focused spots of the hartmannogram. 


\section{Theory}

Let's consider formation of the speckle field by using the optical setup schematically shown in Fig. 2.

The optical field $E_{0}$ is transformed by the diffuser $\mathrm{D}$ and $4 \mathrm{~F}$ transfer system L1-L2 (with the spatial filter A in the confocal plane). The aperture $\mathrm{A}$ is used to control the transfer function of $4 \mathrm{~F}$ system, therefore it could be used to control correlation parameters of the transferred optical field. Intensity distribution in the focal plane of the lenslet array is registered by a CCD-photodetector. Here and in what follows, $\xi$ is the coordinate in the input plane, $\xi^{\prime}$ - coordinate in plane of LA (conjugated with the input plane by the transfer system L1-L2), $\zeta-$ coordinate in plane of the aperture A. Let us consider the phase diffuser with the transmission function:

$t(\xi)=e^{i \varphi(\xi)}$,

where correlation properties of $\varphi(\xi)$ is defined by the dispersion $\sigma_{\varphi}$, correlation radius $\rho_{0}$ and correlation coefficient $\gamma_{\varphi}$. Then, the field behind the diffuser is given as:

$$
E(\xi)=E_{0} e^{i \varphi(\xi)}
$$

In a general case, the transmission in 4F-system is characterized by double Fourier transform with account of the aperture transmission function [6]:

$$
\begin{aligned}
& E_{F}\left(\xi^{\prime}\right)=\hat{F}[E(\zeta)]=\hat{F}[\hat{F}[E(\xi)]]= \\
& =c \int_{-\infty}^{+\infty} \int_{-\infty}^{+\infty} E_{0} e^{i \varphi(\xi)} e^{-\frac{i k \zeta \xi^{\prime}}{F}} P(\zeta) e^{-\frac{i k \xi^{\prime} \zeta}{F}} d \xi d \zeta,
\end{aligned}
$$

where $\hat{F}$ denotes Fourier transform, $k=2 \pi / \lambda$ is the wave vector, $F$ - focal length of lenses in the $4 \mathrm{~F}$ system, $P(\zeta)$ - aperture function. Let's use the spatial frequency

$$
\omega=\frac{k \zeta}{F}
$$

then, (3) can be expressed as:

$$
E_{F}\left(\xi^{\prime}\right)=c e^{\frac{i k \xi^{\prime 2}}{2 F}} \int_{-\infty}^{+\infty} E_{0} e^{i \varphi(\xi)} \int_{-\infty}^{+\infty} P(\omega) e^{-i \omega\left(\xi+\xi^{\prime}\right)} d \xi d \omega
$$

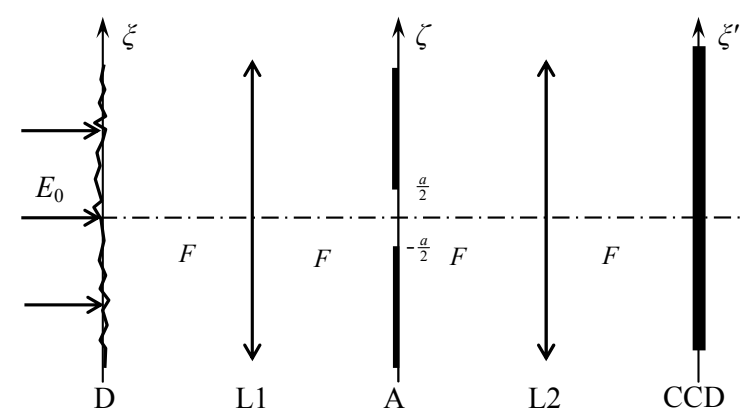

Fig. 2. Schematic diagram of optical setup for wavefront sensing in a speckle field. where $c^{2}=F / i k \lambda$. The correlation function that defines intensity distribution for (3) is obtained as:

$$
\begin{aligned}
& G\left(\xi_{1}^{\prime}, \xi_{2}^{\prime}\right)=\left\langle E_{F}^{*}\left(\xi_{1}^{\prime}\right) E_{F}\left(\xi_{2}^{\prime}\right)\right\rangle \sim\left\langle e^{-i \varphi\left(\xi_{1}^{\prime}\right)} e^{i \varphi\left(\xi_{2}^{\prime}\right)}\right\rangle \approx \\
& \approx e^{-\sigma_{\varphi}^{2}\left(1-\gamma\left(\xi_{1}^{\prime}-\xi_{2}^{\prime}\right)\right)} .
\end{aligned}
$$

For statistically homogeneous Gaussian field, both for a weak $\left(\sigma_{\varphi}^{2}<<1\right)$ and strong diffuser $\left(\sigma_{\varphi}^{2}>1\right)$, the correlation function can be approximated as [7]:

$$
\begin{aligned}
& G\left(\xi_{1}, \xi_{2}\right)=e^{-\sigma_{\varphi}^{2}\left(1-\gamma\left(\xi_{1}-\xi_{2}\right)\right)} \approx \\
& \approx e^{-\sigma_{\varphi}^{2}}+\left(1-e^{-\sigma_{\varphi}^{2}}\right) e^{-\frac{\left(\xi_{1}-\xi_{2}\right)^{2}}{2 \rho_{0}^{2}}}, \\
& \rho_{0}^{2}=\frac{1-e^{-\sigma_{\varphi}^{2}}}{\sigma_{\varphi}^{2}} \rho_{k}^{2},
\end{aligned}
$$

where $\rho_{k}$ is the effective correlation radius $\left(\rho_{k} \approx \rho_{0}\right.$ in the case of weak diffuser). Analyzing the field in the $\xi^{\prime}$ plane, one can see that the speckled image is defined by a "smoothed" diffuser $t_{e}(\xi$ '). The transmission function of "smoothed" diffuser is defined by convolution of real transmission (1) and spatial spectrum of the aperture of 4F system $\widetilde{P}\left(\xi^{\prime}\right)$ :

$t_{e}\left(\xi^{\prime}\right)=\widetilde{P}\left(\xi^{\prime}\right) * t\left(\xi^{\prime}\right)=\int_{-\infty}^{+\infty} \widetilde{P}(z) e^{i \varphi\left(\xi^{\prime}-z\right)} d z$.

Then, the correlation function (7) will be expressed as:

$$
\begin{aligned}
& G_{1}\left(\xi_{1}^{\prime}, \xi_{2}^{\prime}\right)= \\
& =\int_{-\infty}^{+\infty} d z_{1} \int_{-\infty}^{+\infty} \widetilde{P}\left(z_{1}\right) \widetilde{P}\left(z_{2}\right)\left\langle e^{i \varphi\left(\xi_{1}^{\prime}-z_{1}\right)} e^{i \varphi\left(\xi_{2}^{\prime}-z_{2}\right)}\right\rangle d z_{2}= \\
& =\int_{-\infty}^{+\infty} d z_{1} \int_{-\infty}^{+\infty} \widetilde{P}\left(z_{1}\right) \widetilde{P}\left(z_{2}\right) G_{0}\left(\xi_{1}^{\prime}-z_{1}-\xi_{2}^{\prime}+z_{2}\right) d z_{2}= \\
& =G\left(\xi_{2}^{\prime}-\xi_{1}^{\prime}\right) .
\end{aligned}
$$

The correlation value $G\left(\xi_{1}-\xi_{2}\right)$ in (7) depends on the correlation radius in the diffuser relief $\rho_{0}$, and, according to (10), the correlation value $G\left(\xi_{1}^{\prime}-\xi_{2}^{\prime}\right)$ of optical field, which transferred by $4 \mathrm{~F}$ system, depends on the correlation radius of equivalent diffusive surface of "smoothed" diffuser $\rho_{k}$. Thus, the correlation radius can be changed due to the aperture function.

In the case of Gaussian transmission function:

$P(\omega)=e^{-\frac{\omega^{2}}{2 \omega_{0}^{2}}}$

where $\omega_{0}=k a / F$ corresponds to the effective transmission radius of the binary aperture of a diameter $a$, with the spatial spectrum: 
$\widetilde{P}(\xi)=\sqrt{2 \pi} \omega_{0} e^{-\frac{\xi^{2} \omega_{0}^{2}}{2}}$,

(10) takes the form of:

$$
\begin{aligned}
& G(\rho) \sim \\
& \sim \int_{-\infty}^{+\infty} d z_{1} \int_{-\infty}^{+\infty} \exp \left(-\frac{\omega_{0}^{2}}{2}\left(z_{1}{ }^{2}+z_{2}{ }^{2}\right)\right) \exp \left(\frac{\left(\rho-\left(z_{1}-z_{2}\right)\right)^{2}}{2 \rho_{0}^{2}}\right) d z_{2},
\end{aligned}
$$

where $\xi_{1}-\xi_{2}=\rho$. Simple transformation of the correlation function (13) leads to the following form:

$G(\rho) \sim e^{-\frac{\rho^{2}}{2 \rho_{0}^{2}}} \int_{-\infty}^{+\infty} e^{-v^{2}\left(\frac{2}{\rho_{0}^{2}}+\omega_{0}^{2}\right)+\frac{2 \rho v}{\rho_{0}^{2}}} d v$.

The integral (14) could be transformed into:

$$
\begin{aligned}
& G(\rho)=\frac{\sqrt{\pi} \rho_{0}}{\sqrt{2+\rho_{0}^{2} \omega_{0}^{2}}} \exp \left(-\frac{\rho^{2}}{2 \rho_{0}^{2}}\right) \exp \left(\frac{\rho^{2}}{\rho_{0}^{2}\left(2+\rho_{0}^{2} \omega_{0}^{2}\right)}\right) \sim \\
& \sim \exp \left(-\frac{\rho^{2}}{2} \frac{\omega_{0}^{2} \rho_{0}^{2}}{\rho_{0}^{2}\left(2+\omega_{0}^{2} \rho_{0}^{2}\right)}\right)=\exp \left(-\frac{\rho^{2}}{2 \rho_{e f}^{2}}\right)
\end{aligned}
$$

where the correlation function depends on $\rho$ and the required effective correlation radius $\rho_{e f}$. The required effective correlation radius can be received as:

$$
\rho_{e f}^{2}=\frac{2+\omega_{0}^{2} \rho_{0}^{2}}{\omega_{0}^{2}}=\frac{2}{\omega_{0}^{2}}+\rho_{0}^{2} .
$$

Two cases could be considered:

1) The aperture size is much greater than the phase correlation radius in the diffuser plane $\left(\omega_{0}^{2} \rho_{0}^{2}>>2\right)$. In this case, $\rho_{e f} \cong \rho_{0}$, i.e. the correlation radius of the equivalent diffuse surface does not depend on the aperture size and is defined by the correlation radius of diffuser only.

2) The aperture size is much smaller than the phase correlation radius in the diffuser plane $\left(\omega_{0}^{2} \rho_{0}^{2}<<2\right)$. Then, $\rho_{e f}=\frac{\sqrt{2}}{\omega_{0}}$, i.e. the effective correlation radius $\rho_{e f}$, conversely, does not depend on the correlation radius of the diffusive surface and is defined by the aperture.

The dependence of $\rho_{e f}$ on the aperture diameter $a$ and the initial correlation radius $\rho_{0}$ is shown in Fig. 3. One can see that the decrease in the aperture size leads to the increase in the effective correlation radius; it is obvious in the view of optical filtration. Practically, for the initial correlation radius $\rho_{0} \sim 50 \mu \mathrm{m}$ the condition $\omega_{0}^{2} \rho_{0}^{2} \gg 2$ holds with the aperture size $a=1 \mathrm{~mm}$.

It should be mentioned that usually the aperture transmission is the binary function:

$$
P(\omega)=\operatorname{rect}\left(\frac{\omega}{\omega_{0}}\right)=\left\{\begin{array}{l}
1,|\omega| \leq \frac{1}{2} \omega_{0} \\
0,|\omega|>\frac{1}{2} \omega_{0}
\end{array}\right.
$$

with its spatial spectrum $\widetilde{P}\left(\xi^{\prime}\right)=\operatorname{sinc}\left(\frac{\omega_{0} \xi^{\prime}}{2}\right)$. The value of correlation radius can be obtained from (11) and (17) by using computer simulation. It is defined by $e^{-1 / 2}-$ level of the normilized correlation function $G(\rho) / G(0)$ (Fig. 4). The results for apertures (11) and (17) are compared in the table.

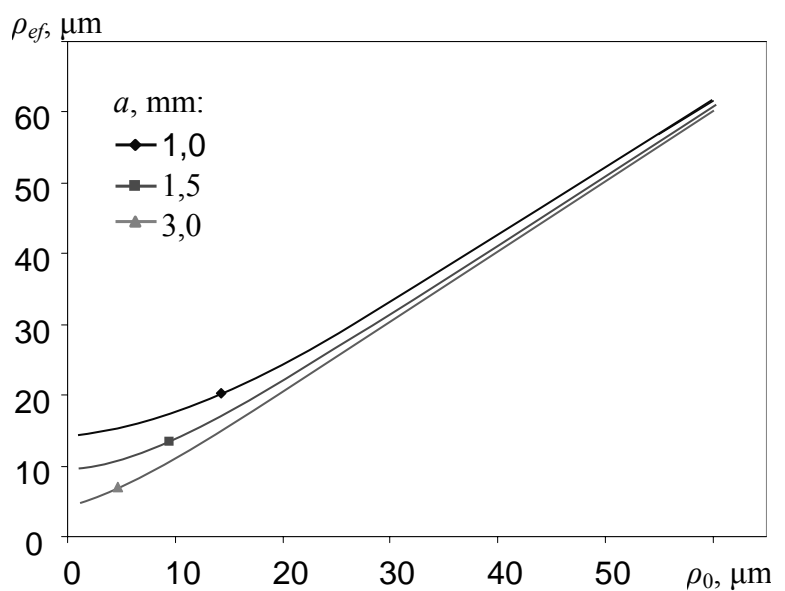

Fig. 3. The dependence of the effective correlation radius $\rho_{e f}$ on the initial correlation radius $\rho_{0}$ for various sizes of the aperture A.

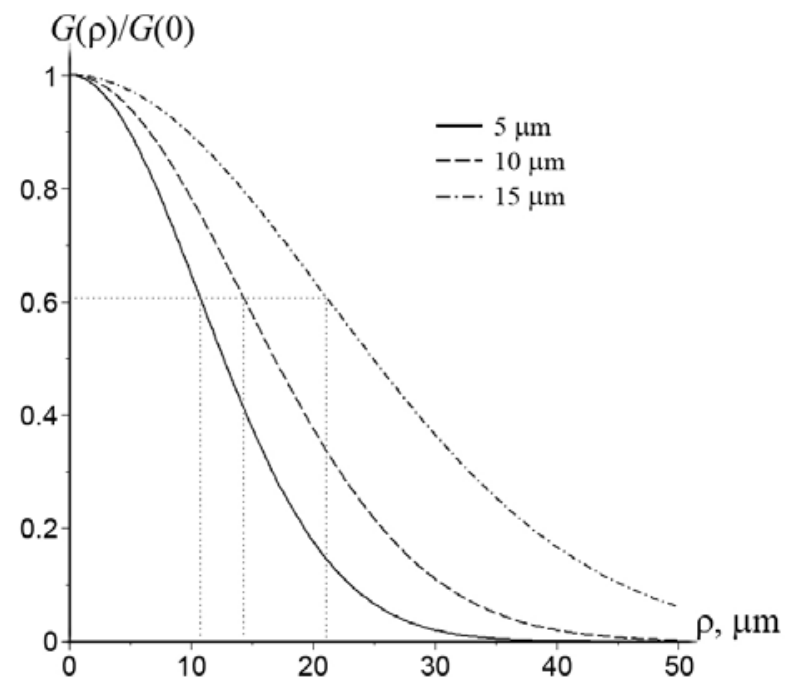

Fig. 4. Normalized correlation function $G(\rho) / G(0)$ for various values of the initial correlation radius $\rho_{0}$ and aperture A size of $1 \mathrm{~mm}$.

Table. Values of the effective correlation radius $\rho_{e f}$ obtained from a rough estimation (11) and exact computer calculation (17).

\begin{tabular}{|c|c|c|}
\hline$\rho_{0}, \mu \mathrm{m}$ & $\rho_{\mathrm{ef}}, \mu \mathrm{m}(11)$ & $\rho_{e f}, \mu \mathrm{m}(17)$ \\
\hline 5 & 15.0 & 10.8 \\
\hline 10 & 17.4 & 14.3 \\
\hline 15 & 20.6 & 21.2 \\
\hline
\end{tabular}




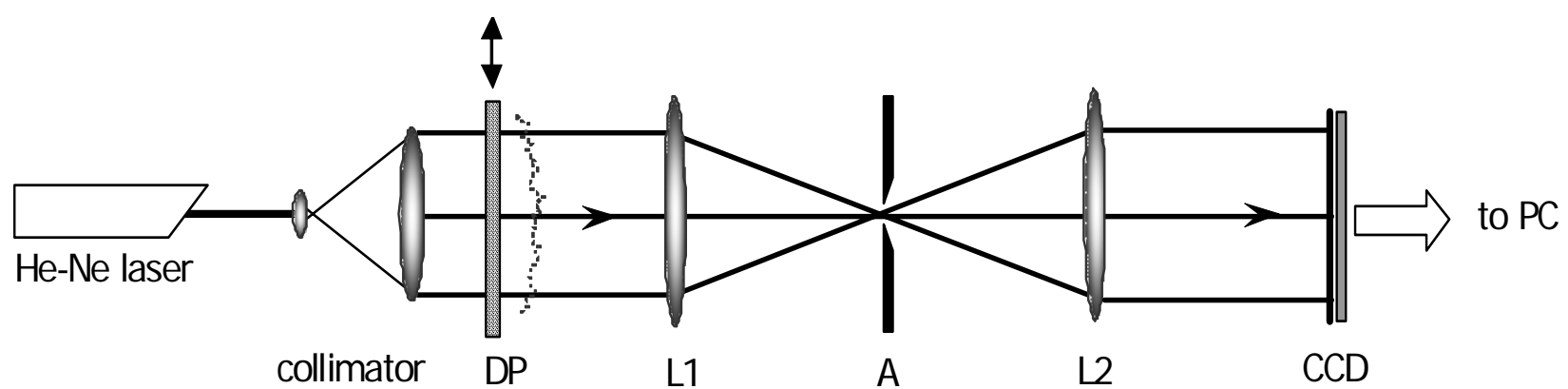

Fig. 5. Optical setup for speckle patterns investigation.

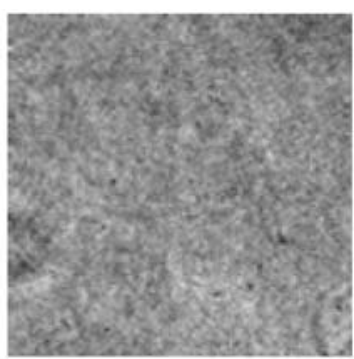

a)

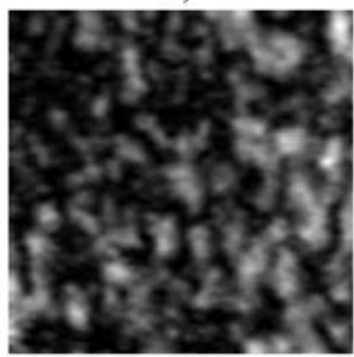

c)

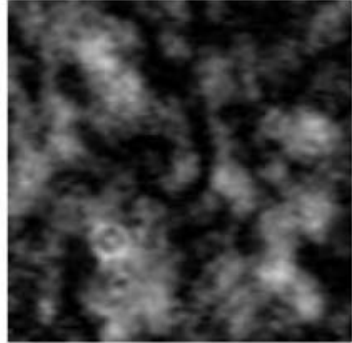

b)

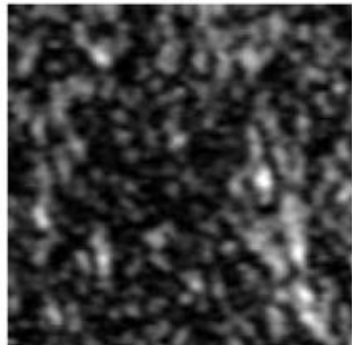

d)

Fig. 6. Speckles for different effective correlation radii of the speckle field: $a$ ) without a speckle; $b) \rho_{e f}$ is $\left.60 \mu \mathrm{m} ; c\right) \rho_{e f}$ is $40 \mu \mathrm{m}$; d) $\rho_{e f}$ is $25 \mu \mathrm{m}$.

One can see that the values of effective correlation radius do not coincide because of different physical meaning of the parameter $\omega_{0}$ in (11) and (17), but $\rho_{e f}$ increases with increasing $\rho_{0}$ in both cases.

\section{Experimental results and discussion}

Experimental setup for investigation of the mentioned phenomenon is shown in Fig. 5. The collimated laser beam passes through the ground-glass diffuse plate DP; the outgoing plane is translated to the measuring plane by means of the telescopic system L2-L3. The statistical parameters of the speckle field in the measuring plane are determined by the ground glass roughness and the diameter of the adjustable spatial filter A. The photodetector CCD captures speckle patterns. Image data from $\mathrm{CCD}$ are processed by the computer PC.

Fig. 6 shows the speckle images for different correlation radii of the speckle field.

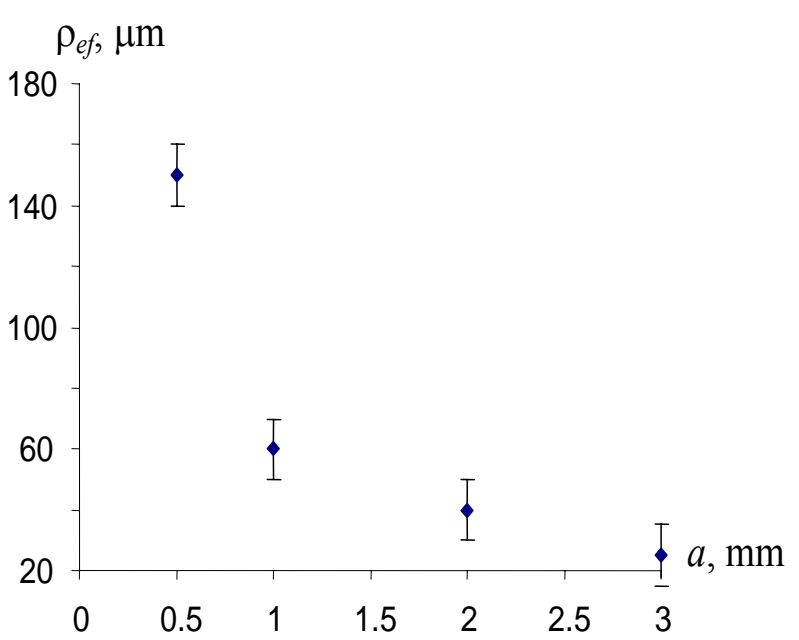

Fig. 7. Measured values of the effective correlation radius in the dependence on a size of the aperture A. 
Since in our investigation we used isotropic media, the speckle patterns were formed only by distribution of the surface heterogeneity. In other words, optical coherent light with an arbitrary wavefront forms the speckle that is equal to the heterogeneity size. The main result of measuring the correlation radius is shown in Fig. 7.

As one can see, the effective correlation radius depends not only on the initial correlation radius of the surface, but also on the aperture of the limited optical system (same as in Fig. 3). Considering the approximations described above, the agreement between the calculated and experimental correlation radii is quite good. We are indeed able to predict the main features of the transferred optical signal, though the calculated correlation radius can be slightly higher than the measured one.

\section{Conclusions}

The proposed model of the optical signal transformation by using the spatially limited optical system has been investigated theoretically and experimentally. The theoretical predictions have been compared with the experimentally obtained dependences of the correlation radius for different apertures. The obtained experimental results have confirmed the theoretical calculations.
References

1. M. Francon, Laser Speckle and Applications in Optics, Academic Press, New York, 1979.

2. R.L. Xu, Particle Characterization Light Scattering Methods, Kluwer Academic Publishers, New York, 2002.

3. V.V. Tuchin, Lasers and Optical Fibers in Biomedical Research. Moscow, Fizmatlit, 2010.

4. D.A. Zimnyakov, V.V. Tuchin, S.R. Utc, The study of the statistical properties of partially developed speckle fields in relation to the diagnosis of structural changes of human skin // Optics and spectroscopy, 5, p. 13-16 (1994).

5. D.V. Podanchuk, V.P. Dan'ko, M.M. Kotov, N.S. Sutyagina, Holographic Shack-Hartmann wavefront sensor for the aberration measurements in the speckle fields // Semiconductor Physics, Quantum Electronics \& Optoelectronics, 11(1), pp. 29-33 (2008).

6. J.W. Goodman, Introduction to Fourier Optics, 2nd ed. McGraw-Hill, New York, 1996.

7. O.I. Barchuck, A.G. Chumakov, V.N. Kurashov, Optical diagnostic of rough surfaces by inhomogeneously polarized laser illumination // Proc. SPIE, 3317, pp. 305-311 (1997). 